\title{
Nonlinear theory of mirror instability near threshold
}

\author{
E.A. Kuznetsov ${ }^{(a, b)}$, T. Passot ${ }^{(c)}$ and P.L. Sulem ${ }^{(c)}$ \\ (a) P.N. Lebedev Physical Institute RAS, 53 Leninsky Ave., 119991 Moscow, Russia \\ ${ }^{(b)}$ L. D. Landau Institute of Theoretical Physics, 2 Kosygin str., 119334 Moscow, Russia \\ (c) CNRS, Observatoire de la Cote d'Azur, PB 4229, 06304 Nice Cedex 4, France
}

\begin{abstract}
An asymptotic model based on a reductive perturbative expansion of the drift kinetic and the Maxwell equations is used to demonstrate that, near the instability threshold, the nonlinear dynamics of mirror modes in a magnetized plasma with anisotropic ion temperatures involves a subcritical bifurcation, leading to the formation of small-scale structures with amplitudes comparable with the ambient magnetic field.
\end{abstract}

PACS: 52.35.Py, 52.25.Xz, 94.30.cj, 94.05.-a

1. In regions of planetary magnetosheaths close to the magnetopause and in the solar wind as well, magnetic structures with a cigar form elongated along the direction of the ambient magnetic field are commonly observed (see e.g. [1, 2]). According to recent observations [3], more than $60 \%$ of such structures are magnetic depressions (holes) associated with maxima of the density and pressure fluctuations. A typical depth of magnetic holes is about $20 \%$ of the mean magnetic field value and can sometimes achieve $50 \%$. The characteristic width of such structures is of the order of a few ion Larmor radii, and they display an aspect ratio of about 7-10. The origin of these structures is not fully understood but they are often viewed as associated with the nonlinear development of the mirror instability, a kinetic instability first predicted by Vedenov and Sagdeev [4] in 1957.

The linear mirror instability has been extensively studied both analytically (see, e.g. [5, 6]), and by means of particle-in-cell (PIC) simulations [7]. This instability develops in a collisionless plasma, when the anisotropy of the ion temperature exceeds the threshold,

$$
T_{\perp} / T_{\|}-1=\beta_{\perp}^{-1} .
$$

Here $\beta_{\perp}=8 \pi p_{\perp} / B^{2}$ (similarly, $\beta_{\|}=8 \pi p_{\|} / B^{2}$ ), where $p_{\perp}$ and $p_{\|}$are perpendicular and parallel plasma pressures respectively. Such conditions can be met under the effect of the plasma compression in front of the magnetopause [8]. As shown in 
[9, 10, 5], the instability is arrested at large $k$ due to finite ion Larmor radius (FLR) effects.

Mirror structures are also observed when the plasma is linearly stable [11, 12, which may be viewed as the signature of a bistability regime. This property was also established in the framework of anisotropic magnetohydrodynamics, using an energetic argument [13]. The aim of the present paper is to demonstrate that the bistability of mirror structures results from a subcritical bifurcation. As well known, for such a bifurcation, non trivial stationary states below threshold are linearly unstable, while above threshold, initially small-amplitude solutions undergo a sharp transition to a large-amplitude state, associated with a blowup behavior within an asymptotic theory. After reviewing the nonlinear theory of the mirror instability, briefly announced in [14], we demonstrate the subcritical character of the bifurcation in three steps: absence of small-amplitude stationary solution above threshold, existence of an unstable branch of solutions below threshold and blowup behavior for the initial value problem above threshold.

The approach is based on a mixed hydrodynamic-kinetic description, assuming a weak nonlinear regime near threshold. Close to threshold, the unstable modes have wavevectors almost perpendicular to the ambient magnetic field $\mathbf{B}$ $\left(k_{z} / k_{\perp} \ll 1\right)$ with $k_{\perp} \rho_{i} \ll 1$, so that the perturbations can be described using a long-wave approximation. The latter allows one to apply the drift kinetic equation (see, e.g., [15, 16]) to estimate the main nonlinear effects that correspond to a local shift of the instability threshold (1). All other nonlinearities connected, for example, with ion inertia are smaller. As the result, we obtain an asymptotic equation for the parallel magnetic field fluctuation, $\widetilde{B_{z}}$ that displays a quadratic nonlinearity. We show that this equation belongs to the generalized gradient type with a free energy that decreases in time, associated with the development of magnetic holes. This process has a self-similar blow-up behavior. This means that possible stabilization of the instability can only take place for amplitudes of order one, a regime that is beyond the framework of the present asymptotics. The present approach contrasts with the quasi-linear theory [17] that also assumes vicinity of the instability threshold but, being based on a random phase approximation, cannot predict the appearance of coherent structures. Phenomenological models based on the cooling of trapped particles were proposed to interpret the existence of deep magnetic holes [18, 19]. These models do not however address the initial value problem in the mirror unstable regime.

2. Consider for the sake of simplicity, a plasma with cold electrons. To describe the mirror instability in the long-wave limit it is enough to use the drift kinetic equation for ions ignoring parallel electric field $E_{\|}$and transverse electric drift:

$$
\frac{\partial f}{\partial t}+v_{\|} \mathbf{b} \cdot \nabla f-\mu \mathbf{b} \cdot \nabla B \frac{\partial f}{\partial v_{\|}}=0 .
$$

In this approximation ions move along the magnetic field $(\mathbf{b}=\mathbf{B} / B)$ due to the magnetic force $\mu \mathbf{b} \cdot \nabla B$ where $\mu=v_{\perp}^{2} /(2 B)$ is the adiabatic invariant which plays 
the role of a parameter in equation (2). Both pressures $p_{\|}$and $p_{\perp}$ are given by

$$
\begin{gathered}
p_{\|}=m B \int v_{\|}^{2} f d \mu d v_{\|} d \varphi \equiv m \int v_{\|}^{2} f d^{3} v, \\
p_{\perp}=m B^{2} \int \mu f d \mu d v_{\|} d \varphi \equiv \frac{1}{2} m \int v_{\perp}^{2} f d^{3} v .
\end{gathered}
$$

Equation (2) with relations (3) and (44) are supplemented with the equation expressing the balance of forces in a plane transverse to the local magnetic field

$$
\begin{aligned}
& \Pi\left\{-\nabla\left(p_{\perp}+\frac{B^{2}}{8 \pi}\right)\right. \\
& \left.+\left[1+\frac{4 \pi}{B^{2}}\left(p_{\perp}-p_{\|}\right)\right] \frac{\mathbf{B} \cdot \nabla \mathbf{B}}{4 \pi}\right\}=0 .
\end{aligned}
$$

Here, consistently with the long-wave approximation, we neglect both the plasma inertia and the non-gyrotropic contributions to the pressure tensor. Furthermore, $\Pi_{i k}=\delta_{i k}-b_{i} b_{k}$ denotes the projection operator in the plane transverse to the local magnetic field. In this equation, the first term describes the action of the magnetic and perpendicular pressures, the second term being responsible for magnetic lines elasticity.

The equation governing the mirror dynamics is then obtained perturbatively by expanding Eqs. (2) and (5). In this approach, the ion pressure tensor elements are computed from the system (2), (5), near a bi-Maxwellian equilibrium state characterized by temperatures $T_{\perp}$ and $T_{\|}$and a constant ambient magnetic field $\mathbf{B}_{\mathbf{0}}$ taken along the $z$-direction.

From Eq. (5) linearized about the background field $\mathbf{B}_{\mathbf{0}}$ by writing $\mathbf{B}=\mathbf{B}_{0}+\widetilde{\mathbf{B}}$ $\left(B_{0} \gg \widetilde{B}\right)$ with $\widetilde{\mathbf{B}} \sim e^{-i \omega t+i \mathbf{k} \cdot \mathbf{r}}$, we have

$$
p_{\perp}^{(1)}+\frac{B_{0} \widetilde{B}_{z}}{4 \pi}=-\frac{k_{z}^{2}}{k_{\perp}^{2}}\left(1+\frac{\beta_{\perp}-\beta_{\|}}{2}\right) \frac{B_{0} \widetilde{B}_{z}}{4 \pi} .
$$

Here $k_{z}$ and $k_{\perp}$ are the projections of the wave vector $\mathbf{k}$, and $p_{\perp}^{(1)}$ is calculated from the linearized drift kinetic equation (2):

$$
\frac{\partial f^{(1)}}{\partial t}+v_{\|} \frac{\partial f^{(1)}}{\partial z}-\mu \frac{\partial \widetilde{B}_{z}}{\partial z} \frac{\partial f^{(0)}}{\partial v_{\|}}=0 .
$$

In Fourier space, this equation has the solution

$$
f^{(1)}=-\frac{\mu \widetilde{B}_{z}}{\omega-k_{z} v_{\|}} k_{z} \frac{\partial f^{(0)}}{\partial v_{\|}} .
$$

The mirror instability is such that $\omega / k_{z} \ll v_{t h \|}=\sqrt{2 T_{\|} / m}$. This means that the ions contributing to the resonance $\omega-k_{z} v_{\|}=0$, correspond to the maximum of the ion distribution function. 
After substituting (17) into the first order term for perpendicular pressure (44) and performing integration, we get

$$
p_{\perp}^{(1)}=\beta_{\perp}\left(1-\frac{\beta_{\perp}}{\beta_{\|}}\right) \frac{B_{0} \widetilde{B}_{z}}{4 \pi}-\frac{i \sqrt{\pi} \omega}{\left|k_{z}\right| v_{t h \|}} \frac{\beta_{\perp}^{2}}{\beta_{\|}} \frac{B_{0} \widetilde{B}_{z}}{4 \pi} .
$$

The first term in (8) is due to the difference between perpendicular and parallel pressures, while the second one accounts for the Landau pole.

Equation (86) together with (6) yield the growth rate for the mirror instability in the drift approximation where FLR corrections are neglected [4]

$$
\gamma=\left|k_{z}\right| v_{t h \|} \frac{\beta_{\|}}{\sqrt{\pi} \beta_{\perp}}\left[\frac{\beta_{\perp}}{\beta_{\|}}-1-\frac{1}{\beta_{\perp}}-\frac{k_{z}^{2}}{k_{\perp}^{2} \beta_{\perp}} \chi\right],
$$

where $\chi=1+\left(\beta_{\perp}-\beta_{\|}\right) / 2$. The instability takes place when $\beta_{\perp} / \beta_{\|}-1>\beta_{\perp}^{-1}$ and, near threshold, develops in quasi-perpendicular directions, making the parallel magnetic perturbation dominant.

As shown in Refs. [9, 10, 5], when the FLR corrections are relevant, the growth rate is modified into

$$
\gamma=\left|k_{z}\right| v_{t h \|} \frac{\beta_{\|} \chi}{\sqrt{\pi} \beta_{\perp}^{2}}\left[\varepsilon-\frac{k_{z}^{2}}{k_{\perp}^{2}}-\frac{3}{4 \chi} k_{\perp}^{2} \rho_{i}^{2}\right]
$$

where $\varepsilon=\beta_{\perp} \chi^{-1}\left(\beta_{\perp} / \beta_{\|}-1-\beta_{\perp}^{-1}\right)$ and the ion Larmor radius $\rho_{i}=v_{t h \perp} / \omega_{c i}$ is defined with the transverse thermal velocity $v_{t h \perp}=\sqrt{2 T_{\perp} / m}$ and the ion gyrofrequency $\omega_{c i}=e B_{0} /(m c)$. This growth rate can be recovered by expanding the general expression given in [5], in the limit of small transverse wavenumbers. It can also be obtained directly from the Vlasov-Maxwell (VM) equations in a long-wave limit which retains non gyrotropic contributions [20]. It is important to note that the expression (10) for $\gamma$ is consistent with the applicability condition $\omega / k_{z} \ll v_{t h \|}$, i.e. when the supercritical parameter $|\varepsilon| \ll 1$. In this case the instability saturation happens at small $k_{\perp} \propto \sqrt{\varepsilon}$ due to FLR and for almost perpendicular direction in a small cone of angles, $k_{z} / k_{\perp} \propto \sqrt{\varepsilon}$. As a result, the growth rate $\gamma \propto \varepsilon^{2}$, so that, when defining new stretched variables by

$$
\begin{aligned}
& k_{z}=\varepsilon K_{z} \rho_{i}^{-1}(2 / \sqrt{3}) \chi^{1 / 2} \\
& k_{\perp}=(2 / \sqrt{3}) \sqrt{\varepsilon} K_{\perp} \rho_{i}^{-1} \chi^{1 / 2} \\
& \gamma=\Gamma(2 / \sqrt{3}) \varepsilon^{2} \Omega\left(\sqrt{\pi} \beta_{\perp}\right)^{-1}\left(\chi \beta_{\|} / \beta_{\perp}\right)^{3 / 2}
\end{aligned}
$$

it takes the form

$$
\Gamma=\left|K_{z}\right|\left(1-K_{z}^{2} / K_{\perp}^{2}-K_{\perp}^{2}\right) .
$$

Hence it is seen that, in the $\left(K_{\perp}-\Theta\right)$ plane $\left(\Theta \equiv K_{z} / K_{\perp}\right)$, the instability takes place inside the unit circle: $\Theta^{2}+K_{\perp}^{2}<1$. The maximum of $\Gamma$ is obtained for $K_{\perp}=1 / 2, \Theta= \pm 1 / 2$ and is equal to $\Gamma_{\max }=1 / 8$. Outside the circle the growth rate becomes negative (in agreement with [10]). 
3. As it follows from (6), in the linear regime, near the instability threshold, the fluctuations of perpendicular and magnetic pressures almost compensate each other (compare with (9)). Therefore, in the nonlinear stage of this instability, we can expect that the main nonlinear contributions come from the second order corrections to the total (perpendicular plus magnetic) pressure, i.e.

$$
p_{\perp}^{(1)}+\frac{B_{0} \widetilde{B}_{z}}{4 \pi}+p_{\perp}^{(2)}+\frac{\widetilde{B}_{z}^{2}}{8 \pi}=-\chi \frac{\partial_{z}^{2}}{\Delta_{\perp}} \frac{B_{0} \widetilde{B}_{z}}{4 \pi} .
$$

This result can be obtained rigorously by means of a multi-scale expansion based on the linear theory scalings (11). For this purpose, we introduce a slow time $T$ and slow coordinates $\mathbf{R}$ in a way consistent with (11), and expand the magnetic field fluctuations as a powers series in $\varepsilon^{1 / 2}$ :

$$
\widetilde{B}_{z}=\varepsilon B_{z}^{(1)}+O\left(\varepsilon^{2}\right), \quad \widetilde{\mathbf{B}}_{\perp}=\varepsilon^{3 / 2} \mathbf{B}_{\perp}^{(3 / 2)}+O\left(\varepsilon^{5 / 2}\right),
$$

where $\mathbf{B}^{(n / 2)}$ are assumed to be functions of $\mathbf{R}$ and $T$. Using these expressions it is easy to establish that quadratic nonlinear terms coming from the expansion of $\Pi$ in (5) as well as from the second term in the r.h.s. of Eq. (13) are small in comparison with the quadratic term originating from the magnetic pressure in Eq. (13). Thus, to get a nonlinear model for mirror dynamics it is enough to find $p_{\perp}^{(2)}$. The expansion (14) induces a corresponding expansion for the distribution function and for both pressures. Defining

$$
\widetilde{p}_{\perp}^{(n)}=\pi m \int v_{\perp}^{2} f^{(n)} v_{\perp} d v_{\perp} d v_{\|},
$$

from (44) we have

$$
p_{\perp}^{(2)}=\left(B_{z}^{(1)} / B_{0}\right)^{2} p_{\perp}^{(0)}+2\left(B_{z}^{(1)} / B_{0}\right) \widetilde{p}_{\perp}^{(1)}+\widetilde{p}_{\perp}^{(2)},
$$

up to an additional contribution proportional to $B_{z}^{(2)}$ that cancels out in the final equation due to the threshold condition.

On the considered time scale, the effect of nonlinear Landau resonance is negligible in the contribution to $f^{(2)}$ that can thus be estimated from the equation

$$
v_{\|} \frac{\partial f^{(2)}}{\partial z}+\left(2 \mu^{2} / v_{t h \|}^{2}\right) B_{z}^{(1)} \frac{\partial B_{z}^{(1)}}{\partial z} \frac{\partial f^{(0)}}{\partial v_{\|}}=0 .
$$

For an equilibrium bi-Maxwellian distribution, we have

$$
f^{(2)}=\left(2 \mu^{2} / v_{t h \|}^{4}\right)\left(B_{z}^{(1)}\right)^{2} f^{(0)}
$$

and thus

$$
p_{\perp}^{(2)}=\left(\beta_{\perp}-4 \beta_{\perp}^{2} / \beta_{\|}+3 \beta_{\perp}^{3} / \beta_{\|}^{2}\right) \frac{\widetilde{B}_{z}^{2}}{8 \pi} .
$$

As a consequence, because of the vicinity to threshold we obtain

$$
p_{\perp}^{(2)}+\frac{\widetilde{B}_{z}^{2}}{8 \pi}=\left(1+\beta_{\perp}^{-1}\right) \frac{3 \widetilde{B}_{z}^{2}}{8 \pi}>0 .
$$


Then rewriting equation (13) using the slow variables (111) and rescaling the amplitude

$$
\widetilde{B}_{z} / B_{0}=\varepsilon 2 \chi \beta_{\perp}\left(1+\beta_{\perp}\right)^{-1} U
$$

we arrive at the equation [14]

$$
\frac{\partial U}{\partial T}=\widehat{K}_{Z}\left[\left(\sigma-\Delta_{\perp}^{-1} \frac{\partial^{2}}{\partial Z^{2}}+\Delta_{\perp}\right) U-3 U^{2}\right]
$$

Here $\sigma= \pm 1$, depending of the positive or negative sign of $\varepsilon, \widehat{K}_{Z}=-\mathcal{H} \partial_{Z}$ is a positive definite operator (whose Fourier transform is $\left|K_{Z}\right|$ ), $\widehat{H}$ is Hilbert transform

$$
\widehat{H} f(Z)=\frac{1}{\pi} V P \int_{-\infty}^{\infty} \frac{f\left(Z^{\prime}\right)}{Z^{\prime}-Z} d Z^{\prime}
$$

As seen from the equation, its linear part reproduces the growth rate (12), in particular, the third term in the r.h.s. accounts for the FLR effect.

Equation (16) simplifies when the spatial variations are limited to a direction making a fixed angle with the ambient magnetic field. After a simple rescaling, one gets

$$
\frac{\partial U}{\partial T}=\widehat{K}_{\Xi}\left[\left(\sigma+\frac{\partial^{2}}{\partial \Xi^{2}}\right) U-3 U^{2}\right],
$$

where $\Xi$ is the coordinate along the direction of variation. This equation can be referred to as a "dissipative Korteveg-de Vries (KdV) equation", since its stationary solutions coincide with those of the usual KdV equation. The presence of the Hilbert transform in Eq. (17) nevertheless leads to a dynamics significantly different from that described by soliton equations.

4. Equation (16) (and its 1D reduction (17) as well) possesses the remarkable property of being of the form

$$
\frac{\partial U}{\partial T}=-\widehat{K}_{z} \frac{\delta F}{\delta U}
$$

where

$$
\begin{aligned}
F & =\int\left[-\frac{\sigma}{2} U^{2}+\frac{1}{2} U \Delta_{\perp}^{-1} \partial_{Z}^{2} U+\frac{1}{2}\left(\nabla_{\perp} U\right)^{2}+U^{3}\right] d \mathbf{R} \\
& \equiv-\sigma N / 2+I_{1} / 2+I_{2} / 2+I_{3}
\end{aligned}
$$

has the meaning of a free energy or a Lyapunov functional. This quantity can only decrease in time, since

$$
\frac{d F}{d t}=\int \frac{\delta F}{\delta U} \frac{\partial U}{\partial t} d \mathbf{R}=-\int \frac{\delta F}{\delta U} \widehat{K}_{z} \frac{\delta F}{\delta U} d \mathbf{R} \leq 0
$$

This derivative can only vanish at the stationary localized solutions, defined by the equation

$$
\frac{\delta F}{\delta U}=\left(\sigma-\Delta_{\perp}^{-1} \frac{\partial^{2}}{\partial Z^{2}}+\Delta_{\perp}\right) U-3 U^{2}=0 .
$$


We now show that non-zero solutions of this equation do not exist above threshold $(\sigma=+1)$. For this aim, following Ref. [21], we establish relations between the integrals $N, I_{1}, I_{2}$ and $I_{3}$, using the fact that solutions of Eq. (20) are stationary points of the functional $F$ (i.e. $\delta F=0$ ). Multiplying Eq. (20) by $U$ and integrating over $\mathbf{R}$ gives the first relation

$$
\sigma N-I_{1}-I_{2}-3 I_{3}=0
$$

Two other relations can be found if one makes the scaling transformations, $Z \rightarrow a Z$, $\mathbf{R}_{\perp} \rightarrow b \mathbf{R}_{\perp}$, under which the free energy (18) becomes a function of two scaling parameters $a$ and $b$

$$
F(a, b)=-\frac{\sigma N}{2} a b^{2}+\frac{I_{1}}{2} b^{4} a^{-1}+\frac{I_{2}}{2} a+I_{3} a b^{2} .
$$

Due to the condition $\delta F=0$, the first derivatives of $F$ at $a=b=1$ have to vanish:

$$
\begin{gathered}
\frac{\partial F}{\partial a}=-\frac{\sigma N}{2}-\frac{I_{1}}{2}+\frac{I_{2}}{2}+I_{3}=0, \\
\frac{\partial F}{\partial b}=-\sigma N+2 I_{1}+2 I_{3}=0 .
\end{gathered}
$$

Hence, after simple algebra, one gets the three relations

$$
I_{1}+\frac{\sigma}{2} N=0, \quad I_{3}=-2 I_{1}, \quad I_{2}=3 I_{1} .
$$

For $\sigma=+1$, the first relation can be satisfied only by the trivial solution $U=$ 0 , because both integrals $I_{1}$ and $N$ are positive definite. In other words, above threshold, nontrivial stationary solutions obeying the prescribed scalings do not exist.

In contrast, below threshold, stationary localized solutions can exist. For these solutions, the free energy is positive and reduces to $F_{s}=N / 2$. Furthermore, $I_{3}=$ $\int U^{3} d^{3} R<0$. which means that the structures have the form of magnetic holes. As stationary points of the functional $F$, these solutions represent saddle points, since the corresponding determinant of second derivatives of $F$ with respect to scaling parameters taken at these solutions, is negative $\left(\partial_{a a} F \partial_{b b} F-\left(\partial_{a b} F\right)^{2}=-2 N^{2}<0\right)$. As a consequence, there exist directions in the eignefunction space, for which the free-energy perturbation is strictly negative, corresponding to linear instability of the associated stationary structure. This is one of the properties for subcritical bifurcations.

For the 1D model (17), the proof of instability of stationary solution $U_{0}=$ $-\frac{1}{2} \operatorname{sech}^{2}(\Xi / 2)$ (which coincides with the KDV soliton) is more complicated than in 3D. The corresponding free energy turns out to have a minimum relatively to the scaling parameter. Therefore, one needs to consider the linearized problem for perturbations $W\left(U=U_{0}+W\right)$, which can be formulated as

$$
\frac{\partial W}{\partial T}=-\widehat{K}_{\Xi} \frac{\delta \tilde{F}}{\delta W}
$$


where $\tilde{F}=\frac{1}{2}\langle W|L| W\rangle$ is the quadratic part of the free energy and

$$
L=1-\frac{\partial^{2}}{\partial \Xi^{2}}+6 U_{0}
$$

is the 1D Schrödinger operator.

It is easily seen that the operator $L$ has one neutral (shift) mode $\psi_{1}=\partial_{\Xi} U_{0}$ $\left(L \partial_{\Xi} U_{0}=0\right)$ associated with invariance by space translation, which has one node. Thus, according the oscillation theorem, $L$ has one negative energy level with $E=-5 / 4<0$, corresponding to the ground state $\psi_{0}=\operatorname{sech}^{3}(\Xi / 2)$ (without nodes), which proves the instability of the stationary solution $U_{0}$ with the growth rate equal $\frac{5}{4}\left\langle\psi_{0}|\widehat{K}| \psi_{0}\right\rangle /\left\langle\psi_{0} \mid \psi_{0}\right\rangle>0$.

As a consequence, starting from general initial conditions, the derivative $d F / d t$ (19) is almost always negative, except for unstable stationary points (zero measure) below threshold. In the nonlinear regime, negativeness of this derivative implies $\int U^{3} d^{3} R<0$, which corresponds to the formation of magnetic holes. Moreover, this nonlinear term (in $F$ ) is responsible for collapse, i.e. formation of singularity in a finite time.

5. In order to characterize the nature of the singularity of Eq. (17), it is convenient to introduce the similarity variables $\xi=\left(T_{0}-T\right)^{-1 / 3} \Xi, \tau=-\log \left(T_{0}-\right.$ $T$ ), and to look for a solution in the form $U=\left(T_{0}-T\right)^{-2 / 3} g(\xi, \tau)$, where $g(\xi, \tau)$ satisfies the equation

$$
\frac{\partial g}{\partial \tau}+\frac{2}{3} g+\frac{\xi}{3} \frac{\partial g}{\partial \xi}=\widehat{K}_{\xi}\left[\frac{\partial^{2} g}{\partial \xi^{2}}-3 g^{2}\right]+e^{-\tau} \widehat{K}_{\xi} g .
$$

As time $T$ approaches $T_{0}(\tau \rightarrow \infty)$, the last term in this equation becomes negligibly small and simultaneously $\partial_{\tau} g \rightarrow 0$ so that asymptotically the equation transforms into

$$
\frac{2}{3} g+\frac{\xi}{3} \frac{d g}{d \xi}=\widehat{K}_{\xi}\left[\frac{d^{2} g}{d \xi^{2}}-3 g^{2}\right] .
$$

For the free energy this means that close to $T_{0}$ the first term $\sim N$ turns out to be much smaller in comparison with all other contributions, in particular with $\int U^{3} d \Xi$.

At large $|\xi|$, that corresponds to the limit $T \rightarrow T_{0}$, the asymptotic solution $\tilde{g}$ of Eq. (21) obeys

$$
2 \widetilde{g}+\xi \frac{d \widetilde{g}}{d \xi}=C \xi^{-2}
$$

where $C=\frac{9}{\pi} \int_{-\infty}^{\infty} g^{2}\left(\xi^{\prime}\right) d \xi^{\prime}>0$, and has the form $\tilde{g}=C \xi^{-2} \log \left|\xi / \xi_{0}\right|$. For $U$, it gives the asymptotic solution

$$
U_{\text {asymp }}=\frac{C}{\Xi^{2}} \log \left|\Xi / \Xi_{0}(t)\right|
$$

with $\Xi_{0}(t)=\left(T_{0}-T\right)^{1 / 3} \xi_{0}$, that, as $T \rightarrow T_{0}$, has an almost time independent tail. For $|\Xi|<\left(T_{0}-T\right)^{1 / 3}\left|\xi_{0}\right|$ the solution is negative and becomes singular as $\Xi$ approaches the origin. 
6. Asymptotically self-similar solutions can also be constructed in three dimensions, when rescaling the longitudinal coordinate by $\left(T_{0}-T\right)^{1 / 2}$, the transverse ones by $\left(T_{0}-T\right)^{1 / 4}$ and the amplitude of the solution by $\left(T_{0}-T\right)^{-1 / 2}$. Existence of a finite time singularity for the initial value problem can be established for initial conditions for which the functional $F$ is negative, when the term involving $\sigma$ can be neglected, an approximation consistent with the dynamics:

$$
F \rightarrow F_{\lim } \equiv \frac{I_{1}}{2}+\frac{I_{2}}{2}+I_{3}
$$

To prove this statement, consider the operator $\widehat{K}_{z}^{-1}$, (inverse of the operator $\widehat{K}_{z}$ ), which is defined on functions obeying $\int U\left(Z, \mathbf{R}_{\perp}\right) d Z=0$, a condition consistent with Eq. (16). Then the time derivative of $F_{\lim }$ can be rewritten through the operator $\widehat{K}_{z}^{-1}$ as follows,

$$
\frac{d F_{\lim }}{d T}=-\int U_{T} \widehat{K}_{z}^{-1} U_{T} d \mathbf{R} \leq 0 .
$$

Consider now the positive definite quantity $\widetilde{N}=\int U \widehat{K}_{z}^{-1} U d \mathbf{R} \geq 0$, whose dynamics is determined by the equation

$$
\frac{d \widetilde{N}}{d T}=-2\left(I_{1}+I_{2}+3 I_{3}\right)=-6 F_{\lim }+I_{1}+I_{2} .
$$

Let $F_{\text {lim }}$ be negative initially, then at $T \geq 0$ the r.h.s. of (24) will be positive, and, as a consequence, $\widetilde{N}$ will be a growing function of time.

Introduce now the new quantity $S=-F_{\text {lim }} / \widetilde{N}$ which is positive definite if $\left.F_{\text {lim }}\right|_{T=0}<0$. The time derivative of $S$ is then defined by means of Eqs. (23) and (24):

$$
\frac{d S}{d T}=-\frac{F_{\lim } \widetilde{N}_{T}}{\widetilde{N}^{2}}+\frac{1}{\widetilde{N}} \int U_{T} \widehat{K}_{z}^{-1} U_{T} d \mathbf{R} .
$$

The second term in the r.h.s. of this equation can be estimated using the CauchyBunyakowsky inequality:

$$
\frac{d \widetilde{N}}{d T}=2 \int U \widehat{K}_{z}^{-1} U_{T} d \mathbf{R} \leq 2 \widetilde{N}^{1 / 2}\left(\int U_{T} \widehat{K}_{z}^{-1} U_{T} d \mathbf{R}\right)^{1 / 2},
$$

that gives

$$
\int U_{T} \widehat{K}_{z}^{-1} U_{T} d \mathbf{R} \geq \widetilde{N}_{T}^{2} /(4 \widetilde{N})
$$

Substituting the obtained estimate into Eq. (25) and taking into account definition (22) for $F_{\text {lim }}$ and Eq. (24) as well, we arrive at the differential inequality for $S$ (compare with [22]):

$$
\frac{d S}{d T} \geq \frac{\widetilde{N}_{T}}{\widetilde{N}^{2}}\left[\frac{\widetilde{N}_{T}}{4}-F_{\lim }\right] \geq 15 S^{2} .
$$

Integrating this first-order differential inequality yields

$$
S \geq \frac{1}{15\left(T_{0}-T\right)} .
$$


Here the collapse time $T_{0}=\left(15 S_{0}\right)^{-1}$ is expressed in terms of the initial value $\left.S\right|_{t=0}=S_{0}$. It is interesting to mention that the time behavior of $S$ given by the estimate (26) coincides with that given by the self-similar asymptotics.

7. In this letter, we have presented an asymptotic description of the nonlinear dynamics of mirror modes near the instability threshold. Below threshold, we have demonstrated the existence of unstable stationary solutions. Differently, above threshold, no stationary solution consistent with the prescribed small-amplitude, long-wavelength scaling can exist. For small-amplitude initial conditions, the time evolution predicted by the asymptotic equation (16) leads to a finite-time singularity. These properties are based on the fact that this equation belongs to a class of generalized gradient systems for which it is possible to introduce a free energy or a Lyapunov functional that decreases in time. In 1D, this model can be referred to as the dissipative KDV equation. The difference with the usual KDV equation is connected with the change of the symplectic operator $\partial / \partial Z$ for the KDV case to the positive definite operator $\widehat{K}_{z}=-\widehat{H} \partial / \partial Z$ for (17). This leads to significant changes in the system dynamics.

The singularity formation as well as the existence of unstable stationary structures below the mirror instability threshold obtained with the asymptotic model, can be viewed as features of a subcritical bifurcation towards a large-amplitude state that cannot be described in the framework of the present analysis. Such an evolution should indeed involve saturation mechanisms that become relevant when the perturbation amplitudes become comparable with the ambient field. We note that recent numerical simulations [23, 20] of the VM equations, using either particle-in-cell codes or an Eulerian description, display the formation of magnetic humps above threshold together with a phenomenon of bistability, associated with the existence of stable large-amplitude magnetic holes both below and above threshold. Due to numerical constraints, these simulations are however performed in regimes that are not sufficiently close to threshold, for the present theory to be applicable.

An important question concerns the relation between the present theory of structure formation and the quasi-linear effects that could compete near threshold. An early-time quasi-linear regime could for example modify the onset of coherent structures and, on the other hand, the development of such structures, can also affect the quasi-linear dynamics.

8. This work was performed in the framework of ISSI team "The effect of ULF turbulence and flow chaotization on plasma energy and mass transfers at the magnetopause". The work of EK was supported by RFBR (grant no. 06-01-00665) and by the French Ministère de l'Enseignement Supérieur et de la Recherche during his visit at the Observatoire de la Côte d'Azur, that of TP and PLS by "Programme National Soleil Terre" of CNRS. 


\section{References}

[1] E.A. Lucek, M.W. Dunlop, T.S. Horbury, A. Balogh, P. Brown, P. Cargill, C. Carr, K.H. Fornacon, E. Georgescun and T. Oddy, Annales Geophysicae, 19, 1421, (2001).

[2] K. Sperveslage, F.M. Neubauer, K. Baumgärtel, and N.F. Ness, Nonlin. Processes Geophys. 7, 191, (2000).

[3] J. Soucek, E. Lucek, and I. Danbouras, J. Geophys. Res. (submitted).

[4] A.A. Vedenov and R.Z. Sagdeev, Plas. Phys. \& Problem of Cont. Therm. React., Vol. III, ed. M.A. Leontovich, 332 (Pergamon Press, NY, 1958).

[5] O.A. Pokhotelov, R.Z. Sagdeev, M.A. Balikhin, and R.A. Treumann, J. Geophys. Res. 109, A09213, (2004).

[6] P. Hellinger, Phys. Plasmas 14, 082105 (2007).

[7] S.P. Gary, J. Geophys. Res. 97 (A6), 8519, (1992).

[8] P. Hellinger and P. Travnicek, Geophys. Res. Lett. 110, A04210, (2005).

[9] A. Hasegawa, Phys. Fluids 12, 2642 ,(1969).

[10] A.N. Hall, J. Plasma Physics 21, 431, (1979).

[11] G. Erdös and A. Balogh, J. Geophys. Res. 101 (A1), 1 (1996).

[12] V. Génot, E. Budnik, C. Jacquey, J. Sauvaud, I. Dandouras, and E. Lucek, AGU Fall Meeting Abstracts 2006, C1412+.

[13] T. Passot, V. Ruban and P.L. Sulem, Phys. Plasmas 13, 102310, (2006).

[14] E.A. Kuznetsov, T. Passot, and P.L. Sulem, Phys. Rev. Lett., 8, 235003 (2007).

[15] D.V. Sivukhin, Voprosy teorii plasmy, Vol.1, pp. 7-97, 1963, ed. M.A. Leontovich, Gosatomizdat, Moscow (in Russian).

[16] R.M. Kulsrud, in: Handbook of Plasma Physics, Eds. M.N. Rosenbluth and R.Z. Sagdeev, Volume 1: Basic Plasma Physics I, edited by A.A. Galeev and R.N. Sudan, 115-145 (1983).

[17] V.D. Shapiro and V.I. Shevchenko, Sov. Phys. JETP 18, 1109, (1964).

[18] M.G. Kivelson and D.S. Southwood, J. Geophys. Res. 101, 17,365 (1996).

[19] F.G.E. Pantellini, J. Geophys. Res. 103, 4789 (1998). 
[20] F. Califano, P. Hellinger, E. Kuznetsov, T. Passot, P.L. Sulem, and P. Travnicek, J. Geophys. Res. (submitted).

[21] E.A. Kuznetsov and S.L. Musher, Sov. Phys. JETP 64, 947 (1986).

[22] S.K. Turitsyn, Phys. Rev. E 47, R13 (1993).

[23] K. Baumgärtel, K. Sauer, and E. Dubinin, Geophys. Res. Lett. 30 (14), 1761 (2003). 